\title{
Aqueous Lubrication with the Molecularly Confined Films of Silicone-Based Amphiphilic Block Copolymer Aggregates
}

Takumi Miyamoto, ${ }^{1}$ Naoyuki Yamazaki, ${ }^{2}$ Shunichi Watanabe, ${ }^{2}$ Shinji Yamada, ${ }^{1, *}$

${ }^{1}$ R\&D - Analytical Science Research, Kao Corporation, 1334 Minato, Wakayama, Wakayama 6408580, Japan

${ }^{2}$ R\&D - Hair Care Products Research, Kao Corporation, 2-1-3 Bunka, Sumida-ku, Tokyo 131-8501, Japan

\section{Corresponding Author}

*Shinji Yamada E-mail: yamada.s@kao.com 


\section{Structural Analysis of Bis-Isobutyl PEG-14/Amodimethicone (BIPA) Copolymer}

GPC Measurement. GPC measurement was performed using refractive index detector equipped with two Shodex K-804L columns at $40{ }^{\circ} \mathrm{C}$ under a flow rate of $1.0 \mathrm{~mL} / \mathrm{min}$. Chloroform was used as an eluent. Lauryl dimethylamine (Farmin DM2098, Kao Corp.) was added to the eluent to prevent the adsorption of cationic components in the sample. Molecular weight was calibrated with standard polystyrene samples.

The GPC elution curve is shown in Figure S1. Two major overlapped peaks were observed. The peak 1 at the elution time of about 16 min was attributed to the main polymer. The peak had a shoulder in the region of high molecular weight, possibly due to the formation of two components with different molecular weight during the synthesis process of the polymer. The peak 2 , whose elution time of about $17.4 \mathrm{~min}$, was estimated to be a PEG-type impurity because of the high sensitivity of PEG (large difference in the refractive index between eluent (chloroform) and PEG). Existence of PEG-type impurity was confirmed by NMR measurement (will be described below). The molecular weight $M_{\mathrm{w}}$ of the main polymer (BIPA copolymer) was estimated to be about 9100 and the polydispersity index $\left(M_{\mathrm{w}} / M_{\mathrm{n}}\right)$ of the polymer was about 1.6.

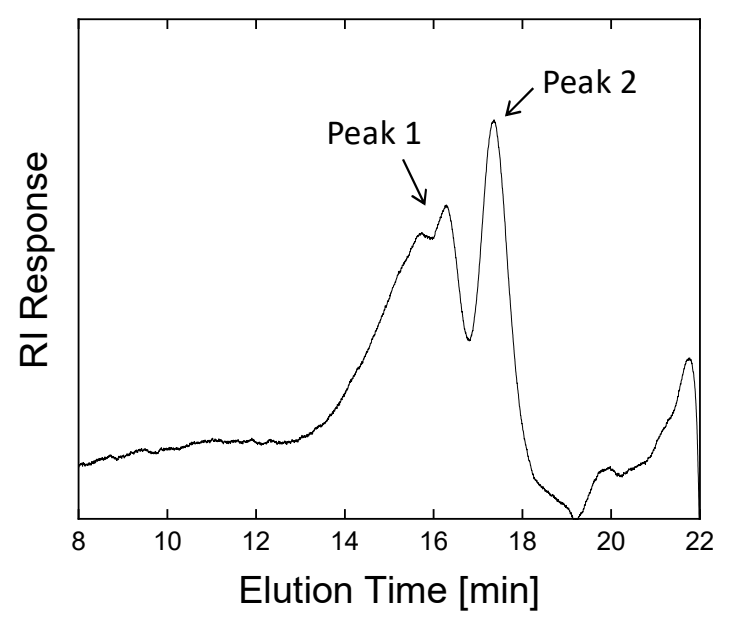

Figure S1. GPC elution curve for BIPA copolymer sample eluted with chloroform at $40^{\circ} \mathrm{C}$.

NMR Measurement. In order to verify the detailed molecular structure of the BIPA copolymer and impurities, ${ }^{1} \mathrm{H}$ NMR spectrum was obtained with a JNM-ECA600 spectrometer operating at $600 \mathrm{MHz}$. 
The sample solution of BIPA copolymer for the ${ }^{1} \mathrm{H}$ NMR measurement was prepared in $\mathrm{CDCl}_{3}$. The obtained ${ }^{1} \mathrm{H}$ NMR spectrum is shown in Fig. S2; peak assignments and peak areas are also included.

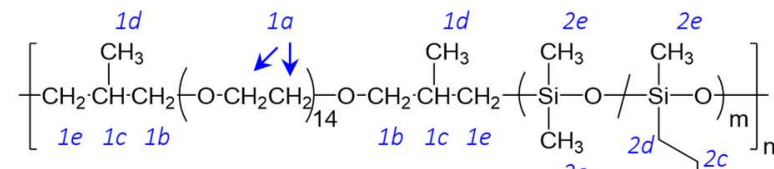

\begin{tabular}{cc}
\hline Peak & Peak Area \\
\hline $1 a$ & 135.69 \\
$1 b$ & 6.24 \\
$1 c$ & 8.46 \\
$1 d$ & 10.00 \\
$1 e$ & 5.98 \\
$2 a$ & 8.17 \\
$2 b$ & 4.49 \\
$2 c$ & 2.84 \\
$2 d$ & 2.64 \\
$2 e$ & 167.87 \\
\hline
\end{tabular}
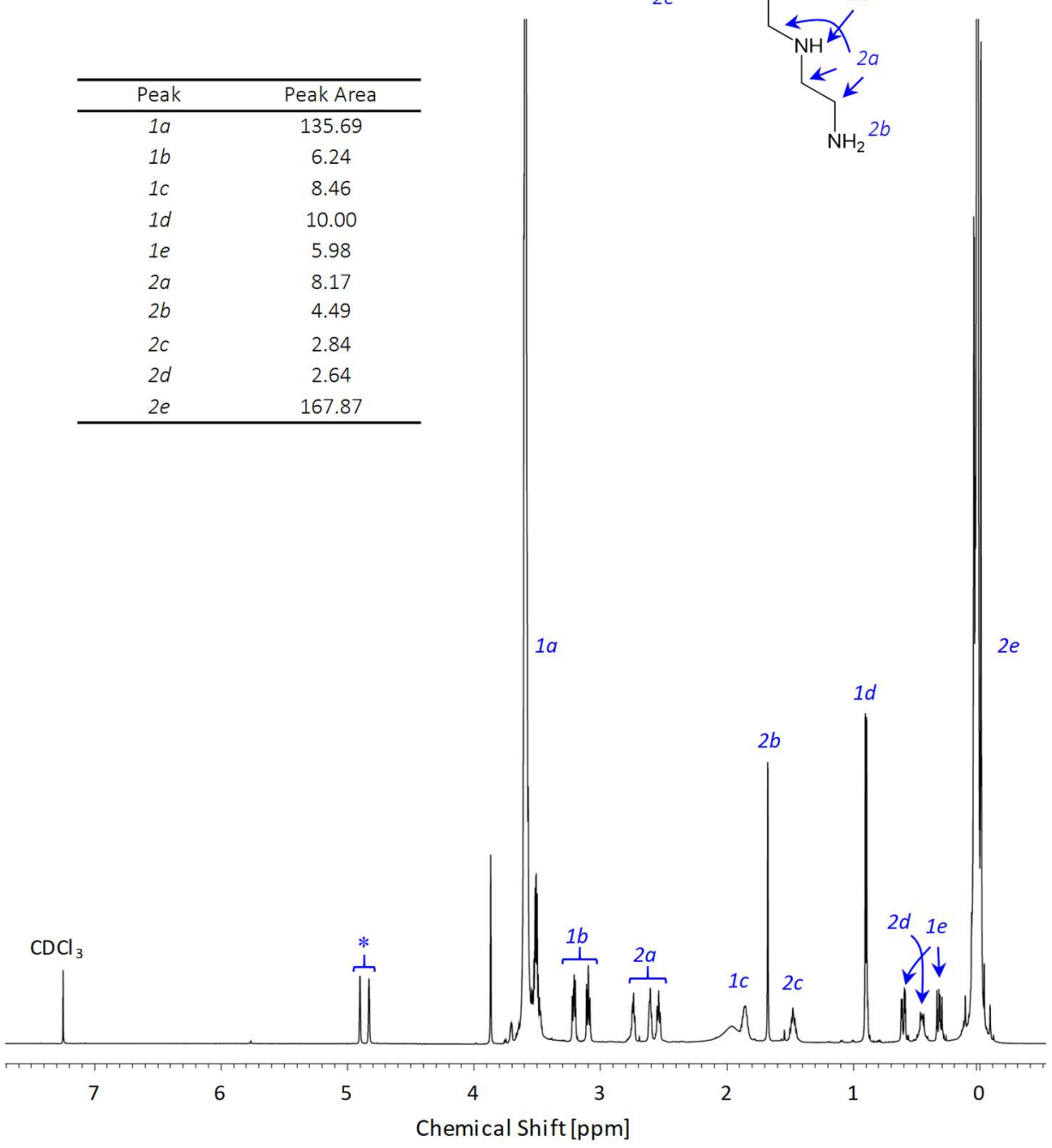

Figure S2. ${ }^{1} \mathrm{H}$ NMR spectrum for the BIPA copolymer sample in $\mathrm{CDCl}_{3}$. Inset table shows the peak area of each peak. Area of peak 1d was set to 10.00 . The peaks marked as asterisk (*) are from the cycrosiloxane impurity.

According to the safety data sheet of this material and the GPC measurement, the sample may include two major impurity components: i) dimethyl, (aminoethylaminopropyl) methyl cycrosiloxanes; and ii) PEG-type component. ${ }^{1}$ H-NMR spectrum shown in Fig. S2 is consistent with this estimation; no 
additional components are expected. Therefore, bipolar gradient stimulated echo (STE) with longitudinal eddy current delay (LED) measurements were also made to quantitatively analyze the impurities in the sample. The peak area $A$ from the specific structures of dimethylsiloxane, aminomodified siloxane, and PEG was fitted by the following equation to separate the contribution of fast and slow components (impurities and main BIPA copolymer)

$$
A=A_{1} e^{-D_{1}\left(\Delta-\frac{\delta}{3}\right)\left(\delta G_{z} \gamma\right)^{2}}+A_{2} e^{-D_{2}\left(\Delta-\frac{\delta}{3}\right)\left(\delta G_{z} \gamma\right)^{2}}
$$

where $D_{1}$ and $D_{2}$ are diffusion coefficients for the fast and slow diffusing populations, $A_{1}$ and $A_{2}$ are the peak area immediately after the $90_{\mathrm{x}}$ pulse, $\Delta$ is the time between the gradient pulses of length $\delta$ and strength $G_{z}$, and $\gamma$ is the gyromagnetic ratio ( for $^{1} \mathrm{H}, \gamma=2.67 \times 10^{8} \mathrm{rad} \mathrm{T}^{-1} \mathrm{~s}^{-1}$ ). Example of the pulsedfield gradient echo attenuation plot is shown in Fig. S3, and the result of the diffusion-coefficient measurement for three specific peaks is listed in Table S1.

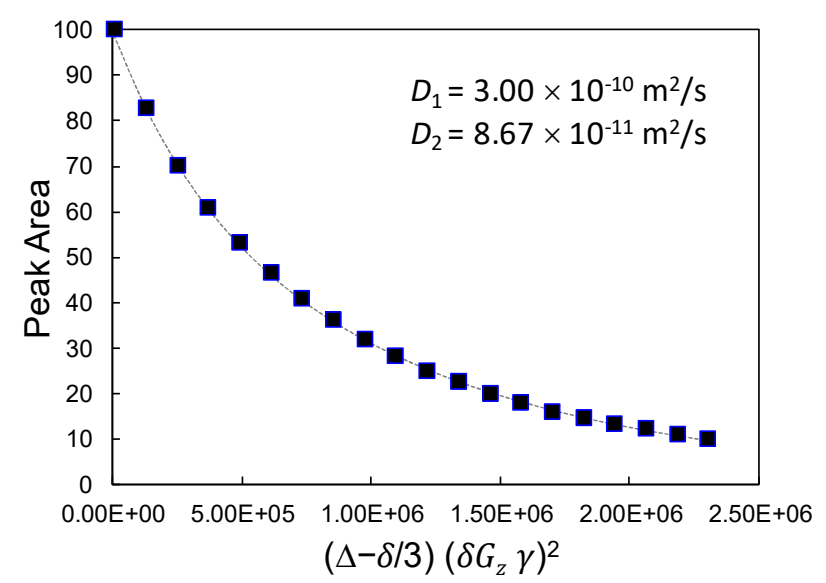

Figure S3. Example of the pulsed-field gradient echo attenuation for the peak from amino-modified siloxane unit (2.538 ppm) in $\mathrm{CDCl}_{3}$. Gray dotted line shows the theoretical fitting using Eq.(1). 
Table S1. Results of the diffusion-coefficient measurement.

\begin{tabular}{|c|c|c|c|c|c|c|}
\hline \multirow[t]{2}{*}{ Peak } & \multicolumn{2}{|c|}{$\begin{array}{c}\text { Dimethylsiloxane unit } \\
(0.017 \text { ppm, assigned as } 2 \mathrm{e} \\
\text { in Fig. S2 })^{\mathrm{a}}\end{array}$} & \multicolumn{2}{|c|}{$\begin{array}{l}\text { Amino-modified siloxane } \\
\text { unit (2.538 ppm, 2a in Fig. } \\
\text { S2) }\end{array}$} & \multicolumn{2}{|c|}{$\begin{array}{c}\text { PEG unit (3.590 ppm, 1a in } \\
\text { Fig. S2) }\end{array}$} \\
\hline & $A[\%]$ & $D\left[\mathrm{~m}^{2} / \mathrm{s}\right]$ & $A[\%]$ & $D\left[\mathrm{~m}^{2} / \mathrm{s}\right]$ & $A[\%]$ & $D\left[\mathrm{~m}^{2} / \mathrm{s}\right]$ \\
\hline Slow & 100 & $1.02 \times 10^{-10}$ & 70.4 & $8.67 \times 10^{-11}$ & 69.4 & $9.60 \times 10^{-11}$ \\
\hline Fast & - & - & 29.6 & $3.00 \times 10^{-10}$ & 30.6 & $2.99 \times 10^{-10}$ \\
\hline
\end{tabular}

${ }^{a}$ This peak was fit by a single exponential decay; fraction from impurity (fast component) was not observed.

Based on the ${ }^{1} \mathrm{H}-\mathrm{NMR}$ spectrum shown in Fig. S2 and diffusion-coefficient measurement listed in Table S1, the detailed molecular structure of the BIPA copolymer and the quantities of impurities were determined as follows:

i) The modification rate of the amino modified siloxane segment was approximately $5 \%$.

ii) The degree of polymerization (DP) of siloxane segments ( $m$ in the molecular structure shown in the inset of Fig. S2) was approximately 16.

iii) On the basis of the GPC measurement $\left(M_{\mathrm{w}} \approx 9100\right)$, the DP of the whole backbone segments ( $\mathrm{n}$ in the molecular structure shown in the inset of Fig. S2) was approximately 5.

iv) The quantity of the cycrosiloxane impurity was approximately $5 \mathrm{wt} \%$, calculated by assuming the chemical structure as hexamethyl, (aminoethylaminopropyl) methyl cyclotetrasiloxane (one of the most thermodynamically stable cycrosiloxane structure ${ }^{1)}$ ).

v) The quantity of the PEG-type impurity was about $12 \mathrm{wt} \%$, calculated as an unreacted bis-isobutyl PEG-14 polymer. 


\section{Structural Characterization of Amphiphilic Block Copolymer Aggregates in Solution}

Hydrodynamic Diameter of BIPA Copolymer Aggregates in Solution. Hydrodynamic diameter of BIPA copolymer aggregates in aqueous solution (concentration of $0.05 \mathrm{wt} \%$ ) was evaluated by means of dynamic light scattering (DLS) using Zetasizer Nano ZS. The result is shown in Fig. S4; the mode diameter was $16 \pm 10 \mathrm{~nm}$.

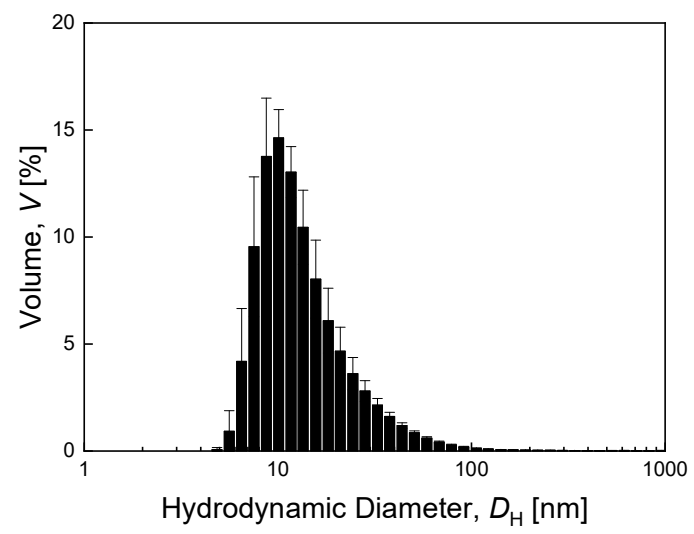

Figure S4. Hydrodynamic diameter distribution of BIPA copolymer aggregates in aqueous solution.

Zeta Potential of BIPA Copolymer Aggregates in Aqueous Solution. Zeta potential of BIPA copolymer aggregates in the solution was measured using Zetasizer Nano ZS for the concentration of $0.05 \mathrm{wt} \%$. The result is shown in Fig. S5; the zeta potential was $47 \pm 7 \mathrm{mV}$.

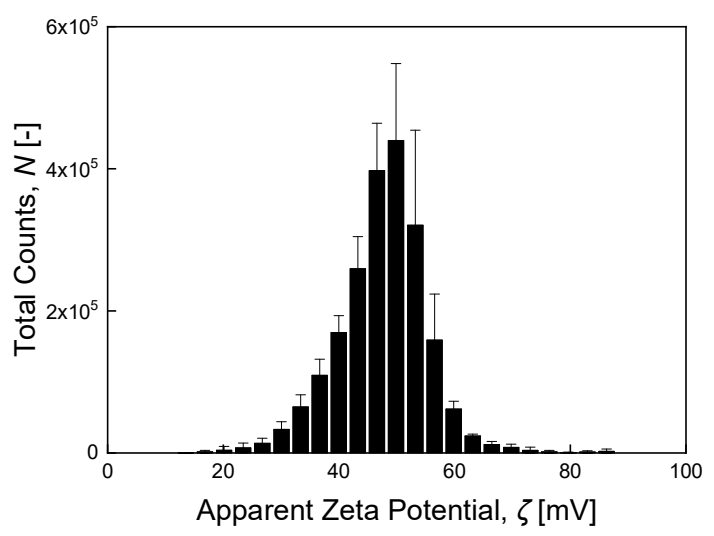

Figure S5. Result of the zeta potential measurement for BIPA copolymer aggregates in aqueous solution. 


\section{Reference}

1) Brown, J. F.; Slusarczuk, G. M. J. Macrocyclic Polydimethylsiloxanes. J. Am. Chem. Soc.1965, 87, 931-932. 